\title{
Hybrid technique to treat superior mesenteric artery occlusion in patients with acute mesenteric ischemia
}

\author{
YAN CHEN $^{1}$, JIECHANG ZHU ${ }^{1}$, ZHICHENG MA ${ }^{2}$, XIANGCHEN DAI $^{1}$, \\ HAILUN FAN $^{1}$, ZHOU FENG ${ }^{1}$, YIWEI ZHANG ${ }^{1}$ and YUDONG LUO ${ }^{1}$ \\ ${ }^{1}$ Department of General Surgery, General Hospital of Tianjin Medical University, Tianjin 300052; \\ ${ }^{2}$ Department of General Surgery, Tianjin First Center Hospital, Tianjin 300192, P.R. China
}

Received September 24, 2014; Accepted January 14, 2015

DOI: $10.3892 / \mathrm{etm} .2015 .2413$

\begin{abstract}
Acute mesenteric ischemia is a condition with a high mortality rate. In the present study, a novel hybrid technique for the treatment of acute mesenteric ischemia was investigated. The retrospective study population included six patients, of which five were male and one was female, with a mean age of 69 years (age range, 59-73 years). The hybrid technique involved isolating the superior mesenteric artery (SMA) for cannulation and subsequently performing a fluoroscopically-assisted embolectomy, retrograde balloon angioplasty and stenting. Intra-arterial, catheter-directed thrombolysis was performed if required. Bowels showing evident necrosis were resected, while ischemic bowels with the potential for recovery were left for $48 \mathrm{~h}$ before being re-examined during the second-look surgery. Retrograde open mesenteric stenting (ROMS) was successfully performed on two patients without bowel resection. Four patients were successfully treated by intra-arterial catheter-directed thrombolysis following recanalization of the SMA, and the ischemic bowels had exhibited a full recovery by the second-look operation. Three patients underwent a massive bowel resection, but did not develop short bowel syndrome. Two patients developed acute renal failure, one of which recovered after 10 days of dialysis, while the other patient succumbed to acute renal failure. In the five surviving patients, the SMA remained patent for the duration of the follow-up period. Therefore, ROMS was shown to be a viable alternative procedure for emergent SMA revascularization. In addition, intra-arterial catheter-directed thrombolysis following recanalization of the SMA was demonstrated as an alternative technique for inhibiting necrosis in bowels with acute mesenteric ischemia.
\end{abstract}

Correspondence to: Dr Yudong Luo, Department of General Surgery, General Hospital of Tianjin Medical University, 154 Anshan Road, Heping, Tianjin 300052, P.R. China E-mail: luoyudong1964@126.com

Key words: catheter-directed thrombolysis, superior mesenteric artery, mesenteric ischemia, thrombosis, embolectomy, angioplasty, stent

\section{Introduction}

Acute mesenteric ischemia is a life-threatening condition with a mortality rate of $40 \%$ (1). The condition is commonly caused by acute thrombosis of a partially occluded atherosclerotic lesion. The perioperative mortality rate of thrombosis in the superior mesenteric artery (SMA) is relatively high due to the difficulty of diagnosis, the extent of bowel ischemia infarction and the complexity of surgical revascularization (2). Treatments for superior mesenteric artery thrombosis include non-surgical treatment (anticoagulant medicines) and surgical treatment, including endarterectomy, artery bypass, artery segmental resection and anastomosis, and intraarterial catheter-directed thrombolysis. During surgery, bowels with necrosis require resection and follow-up surgery is necessary to protect the remaining ischemic bowel tissues (3). Hybrid surgical techniques, a type of surgical treatment combining the advantages of open surgical and endovascular approaches, are widely used and include retrograde open mesenteric stenting (ROMS), which is effective in treating thrombosis in SMA (4). The present study investigated a hybrid approach for treating patients suffering from acute SMA thrombosis, which employed fluoroscopically-assisted embolectomy and balloon angioplasty, combined with catheter-directed thrombolysis during the laparotomy (5).

\section{Materials and methods}

Patients. The hybrid technique was conducted on six patients with acute SMA thrombosis, of which five were male and one was female. Comorbid medical conditions, radiological findings, perioperative data and follow-up information were obtained for all patients (Table I). Three patients presented with sudden and severe abdominal pain, out of proportion with the physical findings, while the other three patients exhibited diffuse, severe and constant pain, with signs of peritoneal irritation. Atherosclerotic lesions were detected using abdominal computed tomography (CT) scans, through which other differential disease diagnoses were able to be excluded. Written informed consent was obtained from the patients. The study was approved by the ethics committee of Tianjin medical university (Tianjin, China).

Surgical procedure. The right common femoral artery was entered percutaneously using the Seldinger technique, and an 
Table I. Medical data of six patients undergoing the hybrid surgery.

\begin{tabular}{lcccccc}
\hline Medical data & Pt 1 & Pt 2 & Pt 3 & Pt 4 & Pt 5 & Pt 6 \\
\hline Gender & M & M & M & F & M & M \\
Age (years) & 59 & 70 & 73 & 72 & 73 & 69 \\
Time to surgery (h) & 15 & 24 & 48 & 24 & 16 & 8 \\
Pain with signs of peritoneal irritation & No & Yes & Yes & Yes & No & No \\
Pre-existing SMA stenosis & Yes & Yes & Yes & Yes & Yes & Yes \\
Atrial tachyarrhythmias & No & No & No & No & No & No \\
Coronary heart disease & No & Yes & Yes & Yes & Yes & No \\
Cerebrovascular disease & No & No & Yes & No & No & No \\
Hypertension & Yes & Yes & Yes & Yes & Yes & Yes \\
Chronic obstructive pulmonary disease & No & No & Yes & Yes & Yes & No \\
\hline
\end{tabular}

Pt, patient; M:F, male:female; SMA, superior mesenteric artery.
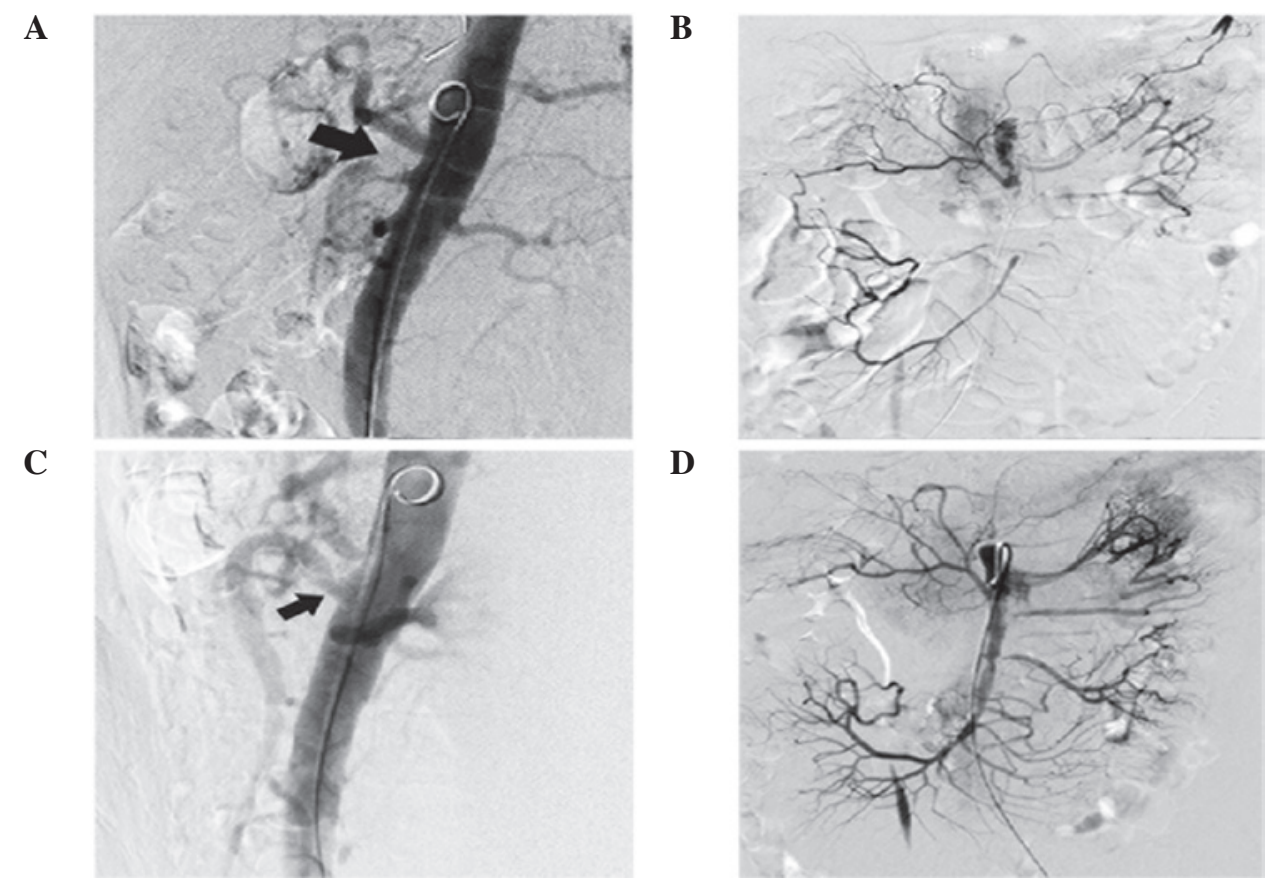

Figure 1. (A) Abdominal aortogram reveals high-grade SMA stenosis at its origin and a distal occluded lesion (arrow identifies the occlusion). (B) Selective angiography of the SMA reveals the distal occlusion with thrombosis. (C) Repeat angiography following the hybrid surgery on the SMA reveals improved visualization of the SMA main trunk. (D) Selective angiography reveals thrombi in the main trunk and distal bed of the SMA. SMA, superior mesenteric artery.

introducer sheath was inserted (6F; Cordis Corporation, Miami Lakes, FL, USA). A pigtail catheter (5F; Cordis Corporation) was inserted into the suprarenal abdominal aorta. An abdominal aorta angiography or selective angiography was conducted for confirmation of thrombosis in the occluded lesion of the SMA (Figs. 1A, 1B and 2A). Subsequently, a midline laparotomy was performed for assessment of the entire intestinal tract. Any clearly necrotic bowel tissue was resected, while ischemic but potentially recoverable tissue was left as much as possible. The SMA was exposed at the root of the mesentery. A puncture (Merit Medical Systems, Inc., South Jordan, UT, USA) was made in the SMA at $\sim 6 \mathrm{~cm}$ distal to the origin, and a $6 \mathrm{~F}$ sheath was inserted in a retrograde fashion. A 0.035 -in Glidewire (Terumo Corporation, Tokyo, Japan) and an angled Glidecatheter (4F; Cordis Corporation) were used to maneuver through the occlusive lesion into the aorta. A 0.018-in control guide wire (Boston Scientific Corporation, Natick, MA, USA) was introduced into the aorta to complete the guide wire exchange. The vessel was clamped distally, a transverse arteriotomy was made at the site of guide wire access and a fluoroscopically-assisted embolectomy was performed using a double-lumen balloon catheter (Edwards Lifesciences Corporation, Irvine, CA, USA) over the wire. A local endarterectomy was conducted if required. A retrograde injection reconfirmed the SMA stenosis. Balloon angioplasty (diameter, 4-5 mm; Medtronic Invatec S.p.A., Roncadelle, Italy) and/or stenting were completed to treat the occlusive lesion. The stent measured $6 \mathrm{~mm}$ in diameter and $40 \mathrm{~mm}$ in length (ev4; Endovasculat, Inc., Plymouth, MN, USA). Following completion of the surgery, the SMA was opened 
A

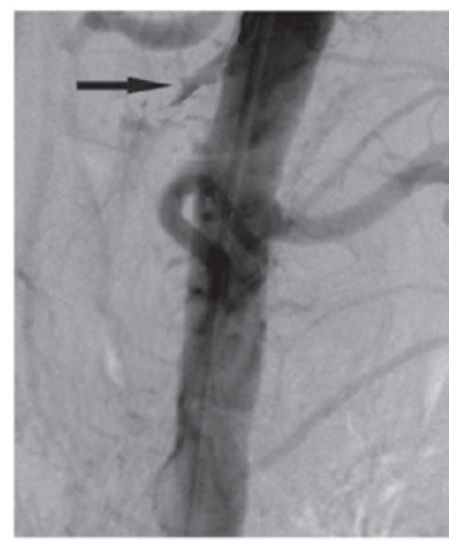

B

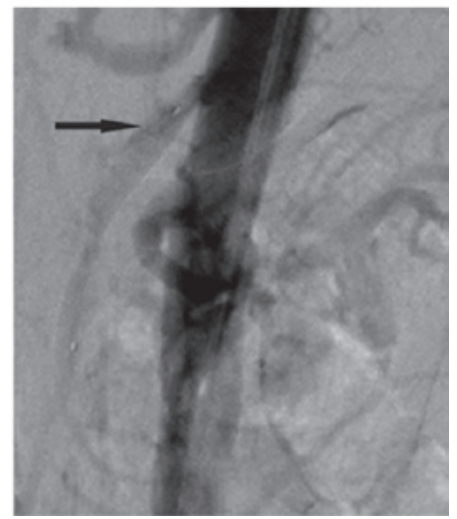

Figure 2. (A) Abdominal aortogram reveals complete SMA occlusion at the origin (arrow identifies the occlusive lesion). (B) A repeat angiography after ROMS reveals the patency of the SMA main trunk (arrow identifies the stent). SMA, superior mesenteric artery; ROMS, retrograde open mesenteric stenting.

to flush out the emboli, the arteriotomy was closed and an angiogram was performed. If blood flow was not restored to the ischemic bowels after $15 \mathrm{~min}$, a catheter $(5 \mathrm{~F} \mathrm{RH}$; Cordis Corporation) was inserted into the SMA for thrombolysis, with urokinase $\left(4 \times 10^{5} \mathrm{IU}\right.$; Biochem Pharmaceutical Corporation, Tianjin, China) administered over $20 \mathrm{~min}$ if necessary. Intra-arterial catheter-directed thrombolysis with urokinase $\left(8 \times 10^{5} \mathrm{IU} / 24 \mathrm{~h}\right)$ was performed continuously for $48 \mathrm{~h}$. In addition, doses of low molecular weight heparin calcium (86 IU/kg, twice a day) were administered to ensure adequate anticoagulation. The safety of the lytic treatment was evaluated every $12 \mathrm{~h}$ by testing the fibrinogen levels (range, 1.80-4.00 g/l). A second-look laparotomy was performed after $48 \mathrm{~h}$ to assess the health outcomes of the bowels, and anastomosis of the bowel segments was performed to restore bowel continuity. Acetylsalicylic acid or warfarin was subsequently administered long term to stabilize the prothrombin time-international normalized ratio (PT-INR) between 2-3 and fibrinogen between $2-4 \mathrm{~g} / \mathrm{L}$. The coagulation function of the patients requires monitoring weekly for at least 1 year and reduced to an appropriate frequency.

Clinical outcomes. Clinical success was defined as the recovery of the ischemic bowels and the resolution of clinical symptoms within the 30-day perioperative period. Clinical examinations, ultrasonography and/or CT-angiography of the SMA were performed to confirm the patency during the follow-up period.

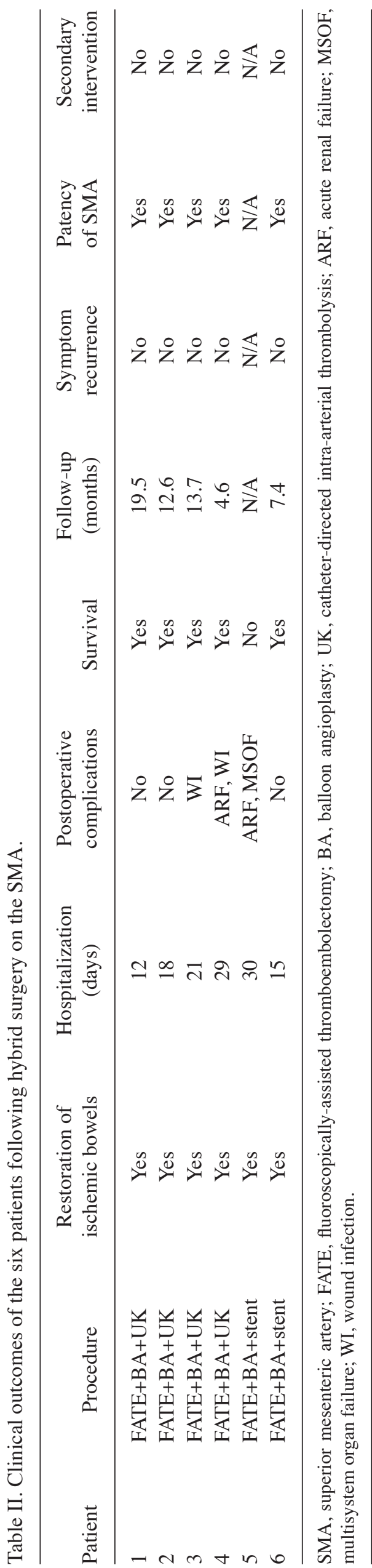




\section{Results}

Stenting. As shown in Fig. 2B, two patients required a single stent, which measured $6 \mathrm{~mm}$ in diameter and $40 \mathrm{~mm}$ in length (ev3 Endovascular, Inc.). These patients experienced a full recovery of the ischemic bowels following revascularization of the SMA during the laparotomy.

Intra-arterial catheter-directed thrombolysis. Four patients required intra-arterial catheter-directed thrombolysis following embolectomy and balloon angioplasty of the SMA (Fig. 1C and 1D). However, one patient was found to have $100 \mathrm{~cm}$ ischemic jejunum tissue following recanalization of the SMA during the first laparotomy. The other three patients exhibited massive bowel necrosis and underwent a necrotic bowel resection during the initial surgery, after which two patients were found to have necrotic small bowels from $80 / 70 \mathrm{~cm}$ proximal jejunum to $40 / 80 \mathrm{~cm}$ terminal ileum, respectively. Although the remaining jejunum was without evident necrosis, the viability of the tissue was poor. The remaining patient exhibited substantial necrosis of the right colon and massive small bowels, with only $90 \mathrm{~cm}$ ischemic proximal jejunum tissue. These four patients underwent intra-arterial catheter-directed thrombolysis for $48 \mathrm{~h}$, and the ischemic bowels were shown to have recovered by the second-look surgery.

Clinical outcomes. Early clinical success was achieved in all the procedures. However, two patients developed acute renal failure postoperatively; one patient recovered after 10 days of dialysis, while the other individual succumbed to acute renal failure. In addition, two patients developed a wound infection. The three patients who underwent massive bowel resection did not develop short bowel syndrome.

Long-term follow-up outcomes. The patency of the SMA was maintained in the five surviving patients during the follow-up period. No patients have experienced recurrence of acute or chronic mesenteric ischemia since the surgical hybrid procedure (Table II).

\section{Discussion}

Acute mesenteric ischemia secondary to a pre-existing SMA atherosclerotic disease is a surgical emergency associated with a poor prognosis. The condition requires prompt diagnosis and early revascularization in order to avoid massive bowel necrosis. Traditional approaches for achieving revascularization involve an antegrade aortomesenteric bypass or retrograde iliac artery to mesenteric artery bypass (3). However, the present study assessed the viability of a combined surgical and endovascular approach, using intraoperative retrograde balloon angioplasty or stenting during the laparotomy, followed by intra-arterial catheter-directed thrombolysis. The application of ROMS enabled the recanalization of the SMA to be more simple and quick, subsequently improving the mortality rate in patients with acute SMA thrombosis.

Milner et al (6) were the first to successfully perform the hybrid ROMS technique on an elderly patient with acute SMA thrombosis. Wyers et al (7) reported a $100 \%$ technical success rate using ROMS on six patients, with a mortality rate of $17 \%$. The mortality rate of ROMS-treated patients was lower when compared with that of conventional surgical revascularization $(80 \%)$ and a percutaneous stent $(100 \%)$ for the treatment of arterial occlusive mesenteric ischemia in 13 patients. Stout et al (8)published a case report that included three patients with acute occlusive mesenteric ischemia who were treated with ROMS, which had a $100 \%$ technical success rate. In the present study, two patients were treated successfully with ROMS, with no technique-related complications or morbidity, although one patient succumbed to acute renal failure.

Previous studies have demonstrated the use of thrombolytic therapy in the treatment of acute mesenteric ischemia (9-11); however, to the best of our knowledge, no studies have applied intra-arterial catheter-directed thrombolysis following recanalization of the SMA. During the laparotomy of the three patients who underwent a massive bowel resection, only 20-30-cm sections of the proximal jejunums were observed to be viable, whereas an ischemic change was evident in the intestinal wall of the remaining proximal jejunum $(50-60 \mathrm{~cm})$. Successful treatment of the $50-60-\mathrm{cm}$ section of the jejunum was necessary for the patients to survive surgery without developing short bowel syndrome. Treating these ischemic bowels was a critical health issue and the only viable treatment strategy was intra-arterial catheter-directed thrombolysis. Although the main trunk of the SMA was patent, a section of the distal vessel bed was occluded with thrombi. Constant intra-arterial catheter-directed thrombolysis may clear the microthrombus and reopen small branches of the SMA to improve the perfusion of the ischemic bowels. Although this thrombolytic therapy presents a risk of hemorrhage for patients simultaneously undergoing an exploratory laparotomy and bowel resection, intra-arterial catheter-directed thrombolysis was an alternative approach for the prevention of necrosis in the ischemic bowels. In the four patients that underwent this procedure in the present study, no bleeding was evident at the time of the thrombolytic therapy. Okamura et al have previously demonstrated that urokinase has no effect during surgery since the half-life in blood is only $16 \mathrm{~min}$ (11). However, in the present study, adjusting the dose of urokinase according to the level of fibrinogen was attempted in order to avoid hemorrhage. Whether catheter-directed thrombolysis in the SMA hybrid surgery should be attempted depends mainly on the viability of the bowels once the SMA has been recanalized.

In conclusion, ROMS was demonstrated to be a viable alternative procedure for emergent SMA revascularization. In addition, intra-arterial catheter-directed thrombolysis following recanalization of the SMA may be an alternative treatment for preventing necrosis in the ischemic bowels of patients with acute mesenteric ischemia. In the future, larger clinical trials are required to investigate the clinical efficacy of the hybrid techniques in the treatment of arterial occlusive mesenteric ischemia.

\section{References}

1. Schoots IG, Koffeman GI, Legemate DA, Levi M and van Gulik TM: Systematic review of survival after acute mesenteric ischaemia according to disease aetiology. Br J Surg 91: $17-27,2004$ 
2. Bayrak S, Bektas H, Duzkoylu Y, Guneyi A and Cakar E: Acute abdomen resulting from concurrent thrombosis of celiac trunk and superior mesenteric artery. Case Rep Gastrointest Med 2014: 142701,2014

3. Matsubara K, Obara H and Kitagawa Y: Diagnosis and treatment of embolism and thrombosis of abdominal aorta and superior mesenteric artery. Nihon Rinsho 72: 1289-1293, 2014.

4. Blauw JT, Meerwaldt R, Brusse-Keizer M, Kolkman JJ, Gerrits D and Geelkerken RH: Retrograde open mesenteric stenting for acute mesenteric ischemia. J Vasc Surg 60: 726-734, 2014.

5. Nomura Y, Yamaguchi M, Kitagawa A, Okada T, Okita Y and Sugimoto K: Hybrid management of ruptured isolated superior mesenteric artery dissecting aneurysm. J Vasc Surg 54: 1808$1811,2014$.

6. Milner R, Woo EY and Carpenter JP: Superior mesenteric artery angioplasty and stenting via a retrograde approach in a patient with bowel ischemia - a case report. Vasc Endovascular Surg 38: 89-91, 2004
7. Wyers MC, Powell RJ, Nolan BW and Cronenwett JL: Retrograde mesenteric stenting during laparotomy for acute occlusive mesenteric ischemia. J Vasc Surg 45: 269-275, 2007.

8. Stout CL, Messerschmidt CA, Leake AE, Veale WN, Stokes GK and Panneton JM: Retrograde open mesenteric stenting for acute mesenteric ischemia is a viable alternative for emergent revascularization. Vasc Endovascular Surg 44: 368-371, 2010.

9. Byun SJ and So BJ: Successful aspiration and thrombolytic therapy for acute superior mesenteric artery occlusion. J Korean Surg Soc 83: 115-118, 2012.

10. Malhotra AD, Chander RK and Kim HS. Catheter-directed thrombolysis for acute superior mesentery artery occlusion: a case report with long-term clinical follow-up. J Vasc Interv Radiol 21: 158-160, 2010.

11. Okamura S, Fujiwara H, Sonoyama T, Ochiai T, Ikoma H, Kubota T, et al: Management of acute superior mesenteric artery occlusion by thrombolytic therapy. Case Rep Gastroenterol 3: 300-305, 2009 